\title{
Análisis de la motivación en el atletismo: un estudio con veteranos*
}

\author{
Analysis of Motivation in Athletics: A Study with Veterans
}

Recibido: julio 5 de 2012 | Revisado: julio 21 de 2013 | Aceptado: octubre 9 de 2013

\author{
ANTONIO ZARAUZ SANCHO ** \\ I.E.S.O. Azcona, España \\ FrANCISCO RUIZ-JUAN *** \\ Universidad de Murcia, España
}

doi:10.11144/Javeriana.UPSY13-2.amae

Para citar este artículo: Zarauz, A. \& Ruiz-Juan, F. (2014). Análisis de la motivación en el atletismo: un estudio con veteranos. Universitas Psychologica, 13(2), 501-515. doi:10.11144/Javeriana.UPSY13-2. amae

* Este trabajo ha sido posible gracias a la inestimable colaboración prestada por D. Fernando Marquina Alonso, Responsable del Departamento de Atletas Veteranos de la Real Federación Española de Atletismo, y por D. Vicente Sánchez Blanco, webmaster de HYPERLINK "http://www.elatleta.com" www.elatleta.com.

** Jefe Departamento Educación Física y Deporte. C/ Santa Laura, 30. 04008. Almería (Almería). Tel: 950-2628 15. E-mail: tonizarauz@msn.com

**** Facultad de Ciencias del Deporte. C/ Argentina, s/n. 30720. Santiago de la Ribera (Murcia). Tel: 868-88 86 75. Fax: 868-88 86 72. E-mail: fruizj@ um.es

\section{RESUMEN}

En la creciente población de atletas veteranos españoles se analiza su motivación desde la perspectiva de la Teoría de la Autodeterminación. Por un lado tratando de establecer relaciones entre ella con la percepción del éxito, la satisfacción intrínseca y la percepción sobre las causas del éxito en el deporte. Por otro, analizando las diferencias en la satisfacción intrínseca, la percepción de éxito y de las creencias sobre las causas del éxito en el deporte y el SDI en función de diversas variables independientes; sexo, edad, hábitos de entrenamiento e historial atlético. Se obtuvieron elevados índices de motivación intrínseca (sobre todo en mujeres), satisfacción, esfuerzo y habilidad, moderados índices de motivación extrínseca, y muy bajos de amotivación, aburrimiento y uso de técnicas de engaño. También se obtienen relaciones positivas y significativas entre la motivación intrínseca con la extrínseca, orientación a la tarea y al ego, diversión y esfuerzo; relaciones positivas y significativas de la motivación extrínseca con la motivación intrínseca, orientación a la tarea y al ego, diversión, aburrimiento, esfuerzo, habilidad y técnicas de engaño; y en amotivación, relaciones positivas y significativas con la motivación extrínseca, aburrimiento y técnicas de engaño, así como negativa con diversión. Por último, del análisis de las variables independientes, se concluye que ser mujer, menor de 50 años, especialista en pruebas de velocidad, salto o lanzamiento, dirigida por un entrenador, que entrena en compañía de otros atletas durante algo más de dos horas por sesión es el perfil motivacional más aconsejable.

Palabras clave

Autodeterminación; Orientación de metas; Adultos; Motivación.

\section{A B S T R A C T}

In the growing population of Spanish master athletes analyzes its motivation from the perspective of Self-Determination Theory. On the one hand trying to establish relations between itself with the perception of success, the intrinsic satisfaction and perception of the causes of success in sport. Furthermore, analyzing the differences in the intrinsic satisfaction, perception of success and beliefs about the causes of success in sport and the SDI based on several independent variables; sex, age, training habits and athletic history. We obtained high levels of intrinsic motivation (especially women), satisfaction, effort and skill, moderate levels of extrinsic motivation, and very low of amotivation, boredom and use of deception techniques. Also obtained positive and significant relationships between intrinsic motivation with extrinsic, task orientation and ego, fun and effort and significant positive relationships of extrinsic motivation to intrinsic motivation, task orientation and ego, fun, boredom, effort, skill and deception techniques, and amotivation, positive and significant relationships with extrinsic motivation, boredom and techniques of deception and negative fun. Finally, analysis of the independent variables, it is concluded to be female, under 50 years, specializing in speed, jump or shoot, directed by a coach, who 
trains in the company of other athletes for over two hours by session is the most advisable motivational profile.

Keywords

Self-determination; Goal orientation; Adults; Motivation.

\section{Introducción}

En el deporte, conducta humana por excelencia, se encuentra la motivación de manera determinante. Por ello, los psicólogos del deporte entran a estudiar y definir la motivación desde esta perspectiva, dada la gran importancia que tiene en el mantenimiento del compromiso o la adherencia a la práctica deportiva. De hecho, Iso-Ahola y St.Clair (2000) llegan a afirmar que la motivación es el mayor y más inmediato determinante del comportamiento humano. Balaguer y Atienza (1994) consideran que la motivación hace referencia a cómo interactúan las variables sociales, ambientales e individuales, determinando la elección de una u otra actividad deportiva, la intensidad de práctica de la misma, la persistencia en la tarea y el rendimiento en ella.

La teoría motivacional más aceptada en la actualidad es la Autodeterminación de Deci y Ryan (1985). En ella, se entiende la motivación como 'un continuo, caracterizada por distintos niveles de autodeterminación que, de mayor a menor, son la motivación intrínseca, la extrínseca y la amotivación'. En el extremo de mayor autodeterminación, se está motivado intrínsecamente al involucrarse en una actividad por propia iniciativa, placer y satisfacción derivados de esta participación, aunque también depende de la percepción de competencia en la especialidad de cada deportista. Por el contrario, se está motivado extrínsecamente al practicar deporte como medio para conseguir un fin y no para su propio beneficio, poniendo como ejemplo a los deportistas que lo hacen para complacer a sus padres o amigos, o simplemente les motiva principalmente conseguir dinero o trofeos. Por último, en el extremo de menor autodeterminación, se explica la amotivación como un estado en que los atletas no están motivados ni intrínseca ni extrínsecamente, simplemente no están motivados, practicando sin propósito, experimentando efectos negativos como apatía, incompetencia, depresión, sin buscar ningún objetivo afectivo, social o material. Pelletier et al. (1995) desarrollaron y validaron un instrumento de medida de estos tres tipos de motivación, la Sport Motivation Scale (SMS), utilizada en diferentes poblaciones de deportistas españoles (Carratalá, 2003; Moreno, Cervelló \& González-Cutre, 2007; Núñez, Martín-Albo \& Navarro, 2007) e incluso en la población de atletas veteranos de pista (De Pero et al., 2009).

En su teoría motivacional de las Metas de Logro, Nicholls (1984) explicó que la meta principal de un sujeto en los contextos de logro es demostrar habilidad, existiendo dos concepciones que se crean por influencia social: la orientación a la tarea (maestría), en la que el éxito viene por el dominio de la tarea y el progreso personal; y la orientación al ego (resultado), en la que el éxito viene por la superación de los rivales y demostración de mayor capacidad que ellos. Por ello, el problema de los deportistas orientados al ego viene al sufrir las primeras derrotas deportivas, pues entonces empiezan a perder su motivación de práctica, al no divertirles perder. Sin embargo, lo opuesto ocurre con los orientados a la tarea, pues al divertirles el simple hecho de la práctica de su especialidad deportiva, su principal objetivo no es ganar en competición, por lo que se suelen mantener en su práctica durante más tiempo que los orientados al ego. Roberts y Balagué $(1989,1991)$ desarrollaron y validaron el Perception of Success Questionaire (POSQ) para medir estas orientaciones motivacionales.

Sin embargo, existe una población en la que poco se ha profundizado en su motivación de práctica deportiva, sobre todo a nivel nacional: los atletas veteranos (mayores de 35 años) que entrenan y compiten habitualmente en pistas de atletismo. Según datos de la Real Federación Española de Atletismo (RFEA, 2012), nuestro país ha pasado de tener 198 licencias federativas de atletas veteranos en 1995, a 5.704 en 2011. El resto de licencias federadas se ha mantenido en estos quince años en algo más de 20.000. Por tanto, el porcentaje de veteranos ha pasado de simbólico a ser casi el $30 \%$. Todos estos datos contrastan con el hecho de que, en España, más del 60\% de los adultos no participa en actividades físico deportivas, el 25\% 
es completamente sedentaria y, además, el 50\% de los adultos que comienzan una actividad física abandona su práctica en los primeros 3 a 6 meses (Moreno, Martínez \& Alonso, 2006). Por esto, el estudio motivacional de veteranos es necesario, a tenor del significativo incremento que ha sufrido en los últimos años.

En esta población de atletas veteranos, parece no existir un consenso claro en las conclusiones obtenidas sobre sus motivaciones, ya se enfoque desde la Teoría de la Autodeterminación o de las Metas de Logro. Da Silva (2009) obtuvo que sus motivaciones eran principalmente intrínsecas, aunque también tenían importancia las extrínsecas. Estudiando las diferencias motivacionales por sexo, mientras que Medic, Starkes, Young y Weir (2006) concluyeron que hombres y mujeres tienen perfiles motivacionales diferentes, siendo mayor la intrínseca en ellas. De Pero et al. (2009) no hallaron diferencias de género, pero sí que a medida que se incrementaba el nivel de competición en los más jóvenes (en una escala que iba de local, a regional, nacional e internacional), cobraba más importancia la motivación extrínseca. Recientemente, RuizJuan y Zarauz (2012a) concluyeron que, tanto en hombres como en mujeres, el entrenamiento y la competición son una parte muy importante en sus vidas, puesto que, además de hallar en ellos una elevada motivación intrínseca y moderada motivación extrínseca, llevan un elevado número de años practicando su especialidad atlética, durante unos cinco días a la semana y dos horas al día, compitiendo frecuentemente a diversos niveles.

Similar disparidad de resultados se encuentran respecto a la percepción del éxito. Así, mientras que Steinberg, Grieve y Glass (2001) obtuvieron que los veteranos mayores de 50 años puntuaban significativamente más bajo que los más jóvenes en la orientación al ego, Medic, Starkes, Young, Weir y Giajnorio (2004) obtuvieron que es a partir de los 65 años donde más alta es la orientación al ego, puesto que es a partir de esa edad cuando disminuye significativamente el número de practicantes y, por consiguiente, cuando menos difícil es conseguir medallas y/o marcas en competición. En cuanto a las diferencias por sexo, Medic, Young, Starkes,
Weir y Grove (2009) obtuvieron que los varones mostraban mayor orientación al ego, al gustarles medirse en competición con los demás, un mayor número de veces al año. Sin embargo, Steinberg et al. (2001) no obtuvieron diferencias significativas respecto a la puntuación en orientación a la tarea con respecto a la edad o el género. Recientemente, Zarauz y Ruiz-Juan (2013a) tampoco obtuvieron diferencias significativas entre sexos en la percepción del éxito de los veteranos, hallando similares valores altos en orientación al ego y a la tarea.

Lo que sí queda más evidenciado, aunque en otras poblaciones de deportistas, son las relaciones entre motivaciones y percepción de las creencias sobre las causas del éxito en el deporte, así como con la satisfacción intrínseca, que está relacionada con la percepción de habilidad y/o capacidad y con la orientación a la tarea (Duda \& Nicholls, 1992). Resultados similares obtuvieron Castillo, Balaguer y Duda (2002) que señalaron que la meta-creenciatarea se asociaba positivamente con la diversión y negativamente con el aburrimiento en el deporte, y la meta-creencia-ego se asociaba positivamente con el aburrimiento. Posteriormente, Castillo, Balaguer, Duda y García-Merita (2004) concluyen que la meta-creencia-tarea predice la participación deportiva a través de la percepción de competencia y la satisfacción intrínseca. Duda, Chi, Newton, Walling y Catley (1995) obtuvieron también una relación positiva y significativa entre la orientación a la tarea y formas de motivación más autodeterminadas.

Por su parte, White, Kavussanu, Tank y Wingate (2004) concluyeron que los atletas percibían que el esfuerzo es lo que lleva al éxito en el deporte y lo relacionaron con la orientación a la tarea, mientras que la orientación al ego la relacionaron con una superior habilidad, factores externos y el empleo de técnicas de engaño. También, Ruiz-Juan, Gómez, Pappous, Alacid y Flores (2010) obtuvieron que la orientación a la tarea estaba relacionada positivamente con el disfrute y que la atribución del éxito en el deporte dependía del esfuerzo. Por último, Zarauz y Ruiz-Juan (2013a) y Ruiz-Juan y Zarauz (2013) obtuvieron que la percepción de éxito por la tarea en atletas veteranos varones de más edad 
se relacionaba con la diversión y autoconfianza, siendo poco importante usar técnicas de engaño.

El objetivo de este estudio es analizar la motivación de los atletas veteranos españoles desde la perspectiva de la Teoría de la Autodeterminación, tratando de establecer relaciones con la percepción del éxito, la satisfacción intrínseca y la percepción sobre las causas del éxito en el deporte. Igualmente, analizar las diferencias en función de diversas variables independientes (sexo, edad, hábitos de entrenamiento e historial atlético). Para ello, se parte de la hipótesis de que las motivaciones de los veteranos españoles son principalmente autodeterminadas y están relacionadas positiva y significativamente con diversión, esfuerzo, ego y tarea.

\section{Método}

\section{Participantes}

Se partió del total de atletas veteranos de pista participantes en competiciones organizadas específicamente para ellos de nivel provincial, autonómico y nacional en España en el año 2011. Según datos de la RFEA (2012), hubo 5.704 licencias federativas de atletas veteranos de pista, de los cuales 4.727 son hombres y 977 son mujeres.

Se obtuvo una muestra aleatoria de 401 atletas veteranos de pista, de los cuales 330 son hombres (82.3\%) con rango de edad de 35 a 75 años $(M=$ $47.98 ; D E=9.14)$ y 71 mujeres $(17.7 \%)$ con rango de edad de 35 a 69 años $(M=45.78 ; D E=10.25)$. Por tanto, se tiene una muestra representativa con un error del $\pm 4.82 \%$, a un intervalo de confianza del $95.5 \%$.

\section{Procedimiento}

Para obtener una muestra lo más amplia y dispersa geográficamente en el territorio español, se pidió colaboración al responsable nacional de atletas veteranos para colgar un enlace en la sección de Veteranos de la Web de la RFEA (http://www.rfea. es/veteranos/veteranos.asp) y así todos los veteranos que visitan la página puedan rellenar el cuestionario telemáticamente. Igualmente, al webmaster del principal foro de atletismo de España (http://www. elatleta.com/foro/forum.php) se le pidió colocar en la sección de atletas veteranos.

En la portada de la web (http://www.retos. org/2encuesta/inicio.html) se informaba el objetivo del estudio, la voluntariedad, la absoluta confidencialidad de las respuestas y manejo de los datos, que no había respuestas correctas o incorrectas y se les solicitó que contestaran con la máxima sinceridad y honestidad. Este trabajo posee informe favorable de la Comisión de Bioética de la Universidad de Murcia.

\section{Instrumentos}

\section{Escala de Motivación Deportiva de Carratalá} (2003); versión española de la Sport Motivation Scale (SMS) de Pelletier et al. (1995)

Fue elaborada para medir los tres tipos de motivación intrínseca (al conocimiento, al logro y a las experiencias estimulantes), los tres tipos de motivación extrínseca (regulación externa, regulación introyectada y regulación identificada) y la amotivación. Consta de 28 ítems distribuidos en siete subescalas, correspondientes a los siete tipos de motivación mencionados, con cuatro ítems cada escala, que son respuestas a la pregunta "iPor qué practica su deporte?", puntuando en escala tipo Likert de 7 puntos desde (1) No se corresponde en absoluto hasta (7) Se corresponde totalmente, con una puntuación intermedia (4) Se corresponde medianamente. Numerosos estudios han mostrado su validez exploratoria y confirmatoria, así como su fiabilidad en el ámbito de la actividad físicodeportiva (Martens \& Webber, 2002; Núñez et al., 2007; Zahariadis, Tsorbatzoudis \& Grouios, 2005), con valores que oscilaron entre $\alpha=0.69$ y $\alpha=0.85$ en las siete subescalas.

\section{Cuestionario de Percepción de Éxito de Cervelló (1996); versión española del Perception of Success Questionnaire (POSQ) de Roberts y Balagué (1989, 1991)}

Fue elaborado para medir la Orientación disposicional de las metas de logro en el contexto deportivo. 
Consta de 12 ítems, 6 de implicación disposicional en la tarea y otras 6 en el ego. La pregunta inicial que encabeza el cuestionario es "Siento éxito en el deporte cuando...". Las respuestas se recogen en escala tipo Likert de que oscilan desde totalmente en desacuerdo (1) a totalmente de acuerdo (5). Estudios previos han mostrado su validez exploratoria y confirmatoria, así como su fiabilidad en el ámbito de la actividad físico-deportiva (Cervelló, Calvo, Ureña, Martínez \& Guzmán, 2006; Cervelló, Escartí \& Balagué, 1999; Cervelló, Hutzler, Reina, Sanz $\&$ Moreno, 2005), con valores $\alpha=0.72$ a $\alpha=0.9$ (tarea) y $\alpha=0.73$ a $\alpha=0.94$ (ego).

Cuestionario de Satisfacción Intrínseca en el Deporte de Balaguer, Atienza, Castillo, Moreno y Duda (1997); versión española del Sport Satisfaction Instrument (SSI) de Duda y Nicholls, (1992)

Fue elaborado para determinar el grado de divertimento. El cuestionario original consta de 8 ítems divididos en dos escalas que miden diversión (5 ítems) y aburrimiento (3 ítems) en la práctica deportiva. Se les pide a los sujetos que indiquen el grado de acuerdo con los ítems, recogiéndose las respuestas en escala tipo Likert que oscila desde (1) muy en desacuerdo a (5) muy de acuerdo. Diversos estudios han mostrado su validez exploratoria y confirmatoria, así como su fiabilidad en el ámbito de la actividad físico-deportiva (Castillo et al., 2002; Castillo et al., 2004; Cervelló et al., 1999; Ruiz-Juan et al., 2010) con valores $\alpha=0.75$ a $\alpha=0.87$ (satisfacción) y $\alpha$ $=0.71$ a $\alpha=0.78$ (aburrimiento).

Inventario de Percepción de las Creencias sobre las Causas del Éxito en el Deporte de Castillo, Balaguer y Duda (2002); versión española del Beliefs About the Causes of Sport Success Questionnaire (BACSSQ) de Duda y Nicholls (1992)

El inventario consta de 18 ítems que miden las percepciones que tienen los sujetos sobre si el esfuerzo (9 ítems, esfuerzo ejercido en el desempeño de la tarea), la habilidad (4 ítems, factores relacionados con la posesión de habilidad) y el uso de técnicas de engaño (5 ítems, utilizar conductas engañosas) permiten alcanzar el éxito en el deporte. En las instrucciones se pregunta a los sujetos: "QQué crees que debería hacer la gente para tener éxito en el deporte que practica más a menudo?", debiendo responder en escala tipo Likert desde (1) muy en desacuerdo a (5) muy de acuerdo. Estudios previos han mostrado su validez exploratoria y confirmatoria, así como su fiabilidad en el ámbito de la actividad físico-deportiva (Cervelló et al., 1999; Guivernau \& Duda, 1994, Ruiz-Juan et al., 2010) con valores $\alpha=0.74$ a $\alpha=$ 0.82 (esfuerzo), $\alpha=0.62$ a $\alpha=0.81$ (habilidad) y $\alpha=0.71$ a $\alpha=0.72$ (técnicas de engaño).

\section{Hábitos de entrenamiento e historial atlético}

Los encuestados respondieron a: ¿Cuál es su especialidad atlética habitual?, ¿A qué nivel ha llegado a competir en su mejor especialidad?, ¿Tiene entrenador?, ¿Cuánto tiempo entrena normalmente al día?, ¿Cuántos días entrena a la semana?, ¿Cuántos años lleva entrenando?, ¿Con cuántos atletas entrena habitualmente?, ¿En cuántas competiciones participa al año?, sexo y edad.

\section{Análisis de los datos}

Análisis de ítems, homogeneidad, consistencia interna (alfa de Cronbach), correlación entre subescalas (coeficiente de Pearson), análisis de varianza y regresión lineal multivariante se realizaron con SPSS 20.0. La estructura factorial se examinó con un análisis factorial confirmatorio (AFC) con AMOS 18.0.

\section{Resultados}

\section{Propiedades psicométricas de los instrumentos}

Para calcular las propiedades psicométricas se siguió el procedimiento de análisis establecido por Carretero-Dios y Pérez (2005). En el análisis de ítems en las cuatro escalas, ningún ítem fue eliminado al cumplir los requisitos establecidos 
(valor $\geq 0.3$ en el coeficiente de correlación corregido ítem-total, desviación estándar $>1$ y todas las opciones de respuesta fueron usadas). El análisis de la homogeneidad indicó la inexistencia de solapamientos de ítems entre las dimensiones teóricas utilizadas en cada una de las cuatro escalas. Se calcularon los índices de asimetría y curtosis siendo, en general, próximos a cero y < 2, tal y como recomiendan Bollen y Long (1994), lo que indica semejanza con la curva normal de forma univariada.

La validez factorial de las cuatro escalas ha sido examinada utilizando el AFC. Se utilizó el "bootstrapping" y el procedimiento de máxima verosimilitud, procedimiento de estimación de modelos de ecuaciones estructurales que asume distribución normal univariada y escala continua de ellos, ya que existe falta de normalidad multivariada en la mayoría de los datos, violando una de las reglas básicas del AFC. El ajuste del modelo fue evaluado con combinación de índices de ajuste absolutos y relativos. Los cuatro modelos presentan valores correctos que permiten determinar una aceptable bondad de ajuste del modelo original (Hoyle, 1995; Hu \& Bentler, 1999; Kline, 1998), como manifiestan los resultados obtenidos (Tabla 1). Los coeficientes estandarizados de relación de la variable latente con cada uno de los ítems, oscilaron entre 0.66 y 0.89 ; en todos los casos las cargas factoriales estandarizadas fueron $>60$ y el $t$-value $>1.96$, lo que garantiza la validez convergente de cada instrumento utilizado en este trabajo (Hair, Black, Babin \& Anderson, 2009).
En la Tabla 2 se presentan los coeficientes alfa de Cronbach y también se incluyen las medias y las desviaciones estándar para todos los atletas. Todas las subescalas demostraron una consistencia interna satisfactoria $(\alpha>0.7)$.

\section{Estadística descriptiva y análisis de correlación}

En la Tabla 2 se observa que la puntuación en motivación intrínseca es mayor que la extrínseca. Los atletas muestran niveles moderadamente altos en motivación intrínseca $(\mathrm{M}=5.2)$, tanto hacia el conocimiento $(M=4.86)$, como al logro $(M=5.1)$ y estimulación $(M=5.62)$. La motivación extrínseca $(\mathrm{M}=3.88)$ arroja niveles moderados, tanto en el factor identificada $(M=4.54)$ como introyectada $(\mathrm{M}=4.23)$, siendo bajas las puntuaciones en regulación externa $(\mathrm{M}=2.87)$ y amotivación $(\mathrm{M}=$ 2.12). Igualmente, como era de esperar por el tipo de colectivo, aburrimiento $(M=1.8)$ y técnicas de engaño $(M=1.41)$ obtienen unas puntuaciones muy bajas. Por el contrario, las puntuaciones en diversión $(M=4.69)$ son muy altas y las obtenidas en esfuerzo $(M=3.44)$ y habilidad $(M=3.27)$ altas. También es alta la percepción de éxito en el deporte por ego $(M=3.14)$ y similar a la tarea $(M=3.17)$.

En la tabla 2, quedan reflejados los resultados de las correlaciones que han sido calculadas. En la motivación intrínseca, se obtuvo una correlación significativa y positiva con motivación extrínseca, orientación a la tarea, orientación al ego, diversión y esfuerzo. En la motivación extrínseca se obtuvieron correlaciones positivas y significativas con motivación intrínseca,

TABLA 1

Índices de ajuste del modelo

\begin{tabular}{lccccccc}
\hline & $\chi^{2} / g l$ & GFI & CFI & IFI & TLI & RMSEA & SRMR \\
\hline Escala de motivación deportiva (SMS) & 2.05 & 0.95 & 0.96 & 0.95 & 0.94 & 0.032 & 0.028 \\
Percepción de éxito en el deporte (POSQ) & 2.61 & 0.96 & 0.95 & 0.96 & 0.95 & 0.042 & 0.044 \\
Satisfacción intrínseca en el deporte (SSI) & 2.89 & 0.95 & 0.94 & 0.94 & 0.94 & 0.042 & 0.039 \\
Percepción de las creencias sobre las causas & 3.88 & 0.94 & 0.93 & 0.93 & 0.92 & 0.051 & 0.045 \\
del éxito en el deporte (BACSSQ) & $<5$ & $>0.9$ & $>0.9$ & $>0.9$ & $>0.9$ & $<0.06$ & $<0.05$ \\
\hline Deseable & $<$
\end{tabular}

Fuente: elaboración propia 


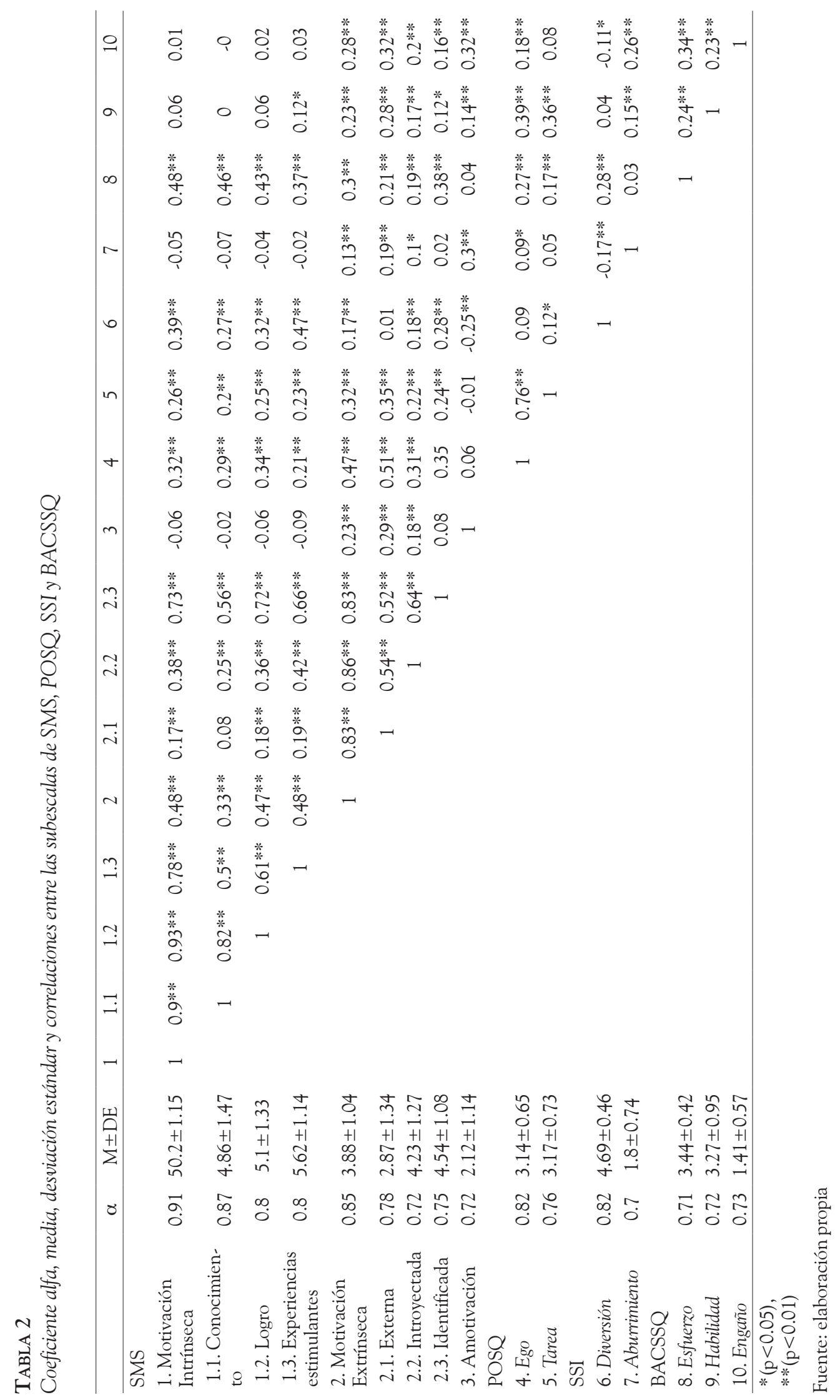


TABLA 3

MANOVA según perfiles de SDI, sexo, edad, especialidad atlética, tener entrenador, minutos de entrenamiento diario y atletas con los que entrena

\begin{tabular}{|c|c|c|c|c|c|c|}
\hline \multirow[t]{2}{*}{ Perfil de SDI } & \multicolumn{2}{|c|}{ Bajo SDI $(N=192)$} & \multicolumn{2}{|c|}{ Alto SDI $(N=209)$} & \multirow[b]{2}{*}{$F$} & \multirow[b]{2}{*}{$p$} \\
\hline & $M$ & $D E$ & $M$ & $D E$ & & \\
\hline Ego & 3.09 & 0.69 & 3.21 & 0.63 & 3.05 & 0.081 \\
\hline Tarea & 3.1 & 0.77 & 3.28 & 0.69 & 6.28 & 0.013 \\
\hline Diversión & 4.5 & 0.56 & 4.86 & 0.26 & 70.61 & 0 \\
\hline Aburrimiento & 1.96 & 0.75 & 1.69 & 0.67 & 14.59 & 0 \\
\hline Esfuerzo & 3.32 & 0.44 & 3.55 & 0.37 & 30.16 & 0 \\
\hline Habilidad & 3.36 & 0.92 & 3.25 & 0.95 & 1.47 & 0.226 \\
\hline Engaño & 1.5 & 0.63 & 1.29 & 0.45 & 14.59 & 0 \\
\hline \multirow[t]{2}{*}{ Sexo } & \multicolumn{2}{|c|}{ Hombres $(N=330)$} & \multicolumn{2}{|c|}{ Mujeres $(N=71)$} & & \\
\hline & $M$ & $D E$ & $M$ & $D E$ & $F$ & $p$ \\
\hline Ego & 3.13 & 0.66 & 3.24 & 0.67 & 1.55 & 0.214 \\
\hline Tarea & 3.19 & 0.72 & 3.23 & 0.81 & 0.15 & 0.693 \\
\hline Diversión & 4.68 & 0.47 & 4.69 & 0.43 & 0.02 & 0.87 \\
\hline Aburrimiento & 1.84 & 0.71 & 1.74 & 0.77 & 1.05 & 0.305 \\
\hline Esfuerzo & 3.42 & 0.41 & 3.51 & 0.43 & 2.44 & 0.119 \\
\hline Habilidad & 3.31 & 0.93 & 3.28 & 0.95 & 0.03 & 0.849 \\
\hline Engaño & 1.38 & 0.52 & 1.45 & 0.69 & 0.84 & 0.358 \\
\hline SDI & 6.83 & 3.88 & 7.68 & 4 & 2.8 & 0.045 \\
\hline \multirow{2}{*}{ Edad } & \multicolumn{2}{|c|}{$<50$ años $(N=249)$} & \multicolumn{2}{|c|}{$>50$ años $(N=152)$} & & \\
\hline & $M$ & $D E$ & $M$ & $D E$ & $F$ & $p$ \\
\hline Ego & 3.19 & 0.61 & 3.09 & 0.72 & 2.43 & 0.119 \\
\hline Tarea & 3.26 & 0.71 & 3.08 & 0.77 & 5.72 & 0.017 \\
\hline Diversión & 4.69 & 0.48 & 4.67 & 0.44 & 0.25 & 0.612 \\
\hline Aburrimiento & 1.88 & 0.72 & 1.72 & 0.71 & 4.66 & 0.031 \\
\hline Esfuerzo & 3.46 & 0.41 & 3.41 & 0.42 & 1.12 & 0.29 \\
\hline Habilidad & 3.35 & 0.93 & 3.22 & 0.93 & 1.83 & 0.177 \\
\hline Engaño & 1.39 & 0.53 & 1.39 & 0.59 & 0 & 0.975 \\
\hline SDI & 7.26 & 3.93 & 6.51 & 3.84 & 3.71 & 0.042 \\
\hline \multirow{2}{*}{ Especialidad atlética } & \multicolumn{2}{|c|}{ Resistencia $(N=190)$} & \multicolumn{2}{|c|}{ Resto $(N=211)$} & & \\
\hline & M & $D E$ & M & $D E$ & F & $p$ \\
\hline Ego & 3.16 & 0.69 & 3.15 & 0.63 & 0.02 & 0.879 \\
\hline Tarea & 3.25 & 0.75 & 3.14 & 0.72 & 2.43 & 0.119 \\
\hline Diversión & 4.66 & 0.51 & 4.71 & 0.42 & 1.10 & 0.293 \\
\hline Aburrimiento & 1.96 & 0.8 & 1.7 & 0.62 & 13.68 & 0 \\
\hline Esfuerzo & 3.45 & 0.43 & 3.43 & 0.4 & 0.22 & 0.638 \\
\hline Habilidad & 3.36 & 0.96 & 3.25 & 0.91 & 1.25 & 0.264 \\
\hline Engaño & 1.43 & 0.63 & 1.36 & 0.47 & 1.62 & 0.203 \\
\hline SDI & 6.65 & 4.27 & 7.28 & 3.54 & 2.56 & 0.11 \\
\hline \multirow[t]{2}{*}{ Tener entrenador } & \multicolumn{2}{|c|}{ SÍ $(N=213)$} & \multicolumn{2}{|c|}{ NO $(N=188)$} & & \\
\hline & $M$ & $D E$ & M & $D E$ & $F$ & $p$ \\
\hline Ego & 3.24 & 0.67 & 3.05 & 0.63 & 7.91 & 0.005 \\
\hline Tarea & 3.28 & 0.75 & 3.09 & 0.71 & 6.68 & 0.01 \\
\hline Diversión & 4.75 & 0.42 & 4.62 & 0.51 & 7.48 & 0.006 \\
\hline Aburrimiento & 1.74 & 0.73 & 1.87 & 0.71 & 1.4 & 0.237 \\
\hline \multirow[t]{2}{*}{ Esfuerzo } & 3.47 & 0.41 & 3.4 & 0.42 & 2.91 & 0.089 \\
\hline & & & & & & Continua \\
\hline 508 & IVERSI' & $\mathrm{CHOLO}$ & $\mathrm{l} \mathrm{V}$ & No. 2 & BRIL-J & | 2014 \\
\hline
\end{tabular}




\begin{tabular}{|c|c|c|c|c|c|c|}
\hline Habilidad & 3.32 & 0.97 & 3.28 & 0.89 & 0.15 & 0.697 \\
\hline Engaño & 1.37 & 0.56 & 1.42 & 0.54 & 0.59 & 0.441 \\
\hline SDI & 7.42 & 3.76 & 6.48 & 4.02 & 5.85 & 0.016 \\
\hline Entrenamiento diario & \multicolumn{2}{|c|}{$<120$ minutos $(N=213)$} & \multicolumn{3}{|c|}{$>120$ minutos $(N=188)$} & \\
\hline & M & $D E$ & M & $D E$ & $F$ & $p$ \\
\hline Ego & 3.08 & 0.63 & 3.23 & 0.68 & 5.03 & 0.025 \\
\hline Tarea & 3.16 & 0.72 & 3.23 & 0.75 & 0.89 & 0.345 \\
\hline Diversión & 4.63 & 0.51 & 4.74 & 0.40 & 5.35 & 0.021 \\
\hline Aburrimiento & 1.86 & 0.71 & 1.78 & 0.73 & 1.34 & 0.246 \\
\hline Esfuerzo & 3.37 & 0.4 & 3.52 & 0.41 & 13.56 & 0 \\
\hline Habilidad & 3.26 & 0.88 & 3.35 & 0.99 & 0.94 & 0.332 \\
\hline Engaño & 1.36 & 0.5 & 1.43 & 0.6 & 1.72 & 0.19 \\
\hline SDI & 6.73 & 3.93 & 7.26 & 3.88 & 1.84 & 0.176 \\
\hline \multirow[t]{2}{*}{ Atletas con los que entrena } & \multicolumn{2}{|c|}{ Solo $(N=136)$} & \multicolumn{2}{|c|}{ Acompañado $(N=265)$} & & \\
\hline & M & $D E$ & $M$ & $D E$ & $F$ & $p$ \\
\hline Ego & 3.16 & 0.65 & 3.15 & 0.66 & 0 & 0.957 \\
\hline Tarea & 3.22 & 0.73 & 3.18 & 0.74 & 0.18 & 0.669 \\
\hline Diversión & 4.63 & 0.56 & 4.71 & 0.41 & 3.97 & 0.045 \\
\hline Aburrimiento & 1.86 & 0.76 & 1.8 & 0.7 & 0.655 & 0.419 \\
\hline Esfuerzo & 3.38 & 0.42 & 3.47 & 0.41 & 4.1 & 0.044 \\
\hline Habilidad & 3.25 & 0.94 & 3.3 & 0.93 & 0.64 & 0.423 \\
\hline Engaño & 1.41 & 0.57 & 1.3 & 0.54 & 0.14 & 0.704 \\
\hline SDI & 6.67 & 4.09 & 7.14 & 3.81 & 1.31 & 0.252 \\
\hline
\end{tabular}

Fuente: elaboración propia

amotivación, orientación a la tarea, orientación al ego, diversión, aburrimiento, esfuerzo, habilidad y técnicas de engaño. En la amotivación se obtuvo una correlación negativa y significativa con diversión, y positiva y significativa con motivación extrínseca, aburrimiento, habilidad y técnicas de engaño.

\section{Análisis de varianza}

En este apartado se realizan diferentes análisis de varianza que se pueden apreciar en la Tabla 3. En primer lugar, se intentó analizar las diferencias en la satisfacción intrínseca, la percepción de éxito y de las creencias sobre las causas del éxito en el deporte en función del índice de autodeterminación (SDI). Este índice se calcula con la siguiente fórmula: $\{2 \mathrm{x}(\mathrm{MI}$ conocimiento $+\mathrm{MI}$ ejecución+MI estimulación)/3) + Regulación Identificada $\}-\{$ (Regulación Externa+Introyección) $/ 2+$ (2xAmotivación) $\}$ (Vallerand,1997).
Este tipo de índice se ha mostrado como un indicador válido de la autodeterminación en diferentes trabajos (Chantal \& Bernache-Assollant, 2003; Chantal, Robin, Vernat \& Bernache-Assollant, 2005; Kowal \& Fortier, 2000; Losier \& Vallerand, 1994; Moreno, Cervelló \& Cutre-Coll, 2007). En este estudio el índice osciló entre -6.22 y +15.88 $(\mathrm{M}=6.98, \mathrm{DE}=3.91)$. La media de 6.98 fue usada para clasificar a los sujetos en dos perfiles motivacionales, de modo que aquellos que presentaban un índice superior a 6.98 se consideró que tenían un alto SDI y aquellos que revelaban un índice inferior a 6.98 se consideró que tenían un bajo SDI.

Los resultados obtenidos, muestran diferencias significativas para orientación a la tarea $(F=$ $6.28, p<0.05)$, diversión $(F=70.61, p<0.05)$, aburrimiento $(F=14.59, p<0.05)$, esfuerzo $(F=$ $30.16, p<0.05)$ y engaño $(F=14.59, p<0.05)$. En este sentido, los atletas con alto SDI muestran mayor orientación a la tarea $(M=3.28)$, diversión $(M=4.86)$ y esfuerzo $(M=3.55)$ que los 
deportistas con bajo SDI $(\mathrm{M}=3.1, \mathrm{M}=4.5, \mathrm{M}$ =3.32). Mientras que estos revelan mayor aburrimiento $(M=1.96)$ y uso de técnicas de engaño $(\mathrm{M}=1.5)$ que los deportistas con alto SDI $(\mathrm{M}=$ $1.69, \mathrm{M}=1.29$ ).

A continuación, se analizaron las diferencias en la satisfacción intrínseca, la percepción de éxito y las creencias sobre las causas del éxito en el deporte y el SDI en función de las variables independientes: sexo del atleta, edad, especialidad atlética, nivel de competición alcanzado, tener entrenador, tiempo de entrenamiento diario, días de entrenamiento semanales, años de entrenamiento, número de atletas con los que entrena y número de competiciones en las que participa anualmente. El MANOVA calculado mostró diferencias significativas en función de estas variables.

No se encontraron efectos de interacción de segundo orden entre las variables independientes $(p>0.05)$. Tampoco se hallaron diferencias estadísticamente significativas entre las variables dependientes y las variables independientes: nivel de competición alcanzado, días de entrenamiento semanales, años de entrenamiento y número de competiciones en las que participa anualmente.

Sin embargo, sí se detectaron diferencias respecto a los efectos principales en el resto de variables independientes (Tabla 3). Según el sexo de los sujetos, solo se encontraron diferencias significativas en SDI $(F=2.8, p<0.05)$, de tal forma que las mujeres presentan un SDI $(M=7.68)$ mayor que los hombres $(\mathrm{M}=6.83)$.

Según la edad, los resultados muestran diferencias significativas en orientación a la tarea $(F=$ 5.72, $p<0.05)$, aburrimiento $(F=4.66, p<0.05)$ y SDI $(F=3.71, p<0.05)$, revelando que los atletas de menor edad ( $<50$ años) tienen una mayor orientación a la tarea $(M=3.26)$, aburrimiento $(M$ $=1.88)$ y SDI $(M=7.26)$ que los de mayor edad $(>$ 50 años) ( $M=3.08, M=1.72, M=6.51)$.

Atendiendo a la especialidad atlética, solo se encontraron diferencias significativas en aburrimiento $(F=13.68, p<0.05)$, de tal forma que los atletas que compiten en pruebas de resistencia (medio fondo, fondo, obstáculos y marcha) ( $\mathrm{M}=$ 1.96) se aburren más quienes lo hacen en el resto de pruebas (velocidad, vallas, saltos, lanzamientos, pruebas combinadas) $(\mathrm{M}=1.7)$.

En cuanto a tener entrenador, los resultados obtenidos muestran diferencias significativas en orientación al ego $(F=7.91, p<0.05)$, orientación a la tarea $(F=6.68, p<0.05)$, diversión $(F=7.48$, $p<0.05)$ y SDI $(F=5.85, p<0.05)$. En este sentido, los atletas que tienen entrenador revelan una mayor orientación al ego $(M=3.24)$, a la tarea $(M$ $=3.28)$, diversión $(M=4.75)$ y SDI $(M=7.42)$ que los que no tienen entrenador $(M=3.08, M=$ 3.09, $M=4.62, M=6.48$ ).

En el tiempo de entrenamiento diario, se hallaron diferencias significativas en orientación al ego $(F=5.03, p<0.05)$, diversión $(F=5.35, p<0.05)$ y esfuerzo $(F=13.56, p<0.05)$. Así, los atletas que entrenan más de 120 minutos diarios presentan una mayor orientación al ego $(M=3.23)$, diversión ( $M$ $=4.74)$ y esfuerzo $(M=3.52)$ que aquellos que entrenan menos de 120 minutos al día $(M=3.08, M$ $=4.63, \mathrm{M}=3.37$ ).

Finalmente, en el número de atletas con los que se entrena habitualmente, las diferencias significativas se obtuvieron en diversión ( $F=3.97, p<$ $0.05)$ y esfuerzo $(F=4.1, p<0.05)$, siendo los que entrenan acompañados lo que arrojan una mayor diversión $(M=4.71)$ y esfuerzo $(M=3.47)$ que los que entrenan solos $(M=4.63, M=3.38)$.

\section{Análisis regresivo multivariante}

A continuación, se realizó un análisis de regresión lineal multivariado, tratando de obtener un modelo que explicase la mayor parte posible de varianza. Se tomó como variable dependiente la puntuación del índice de autodeterminación (SDI) y como variables predictoras cada una de las variables de la percepción de éxito en el deporte (orientación a la tarea y orientación al ego), la satisfacción intrínseca en el deporte (diversión y aburrimiento) y percepción de las creencias sobre las causas del éxito en el deporte (esfuerzo, habilidad y técnicas de engaño).

Se obtiene un sólido modelo ya que explica más de la mitad de la varianza (57.7\%). Del análisis se extrajo el valor de $R^{2}(0.577)$ para explicar la varianza, el de Beta para explicar la predicción entre 
variables, el de F (32.764) para ver si existe relación entre las variables seleccionadas y su significatividad $(p<0.001)$.

El modelo obtenido muestra que un mayor índice de autodeterminación (SDI) se puede predecir significativamente por puntuar bajo en técnicas de engaño (Beta $=-0.249, p<0.001$ ), aburrimiento $($ Beta $=-0.138, p=0.002)$ y habilidad $($ Beta $=$ $-0.143, p=0.002)$, así mismo por puntuar alto en diversión (Beta $=0.277, p<0.001$ ), esfuerzo (Beta $=0.314, p<0.001$ ) y orientación a la tarea (Beta $=0.121, p=0.007$ ).

\section{Discusión}

Las motivaciones de los veteranos españoles resultaron ser fundamentalmente intrínsecas, sobre todo en mujeres, aunque también tuvieron una importancia moderada las extrínsecas, al igual que obtuvieron Da Silva (2009), Medic et al. (2006), Ruiz-Juan y Zarauz (2012a) y Zarauz y Ruiz-Juan (2013b). Estos altos niveles de autodeterminación probablemente sean la causa de que los atletas veteranos mantengan la adherencia a su especialidad durante tantos años en su vida, como explicaban Baker, Horton y Weir (2010).

Por otra parte, los similares niveles moderados en motivación extrínseca obtenidos por hombres y mujeres, al igual que De Pero et al. (2009) y Ruiz-Juan y Zarauz (2012a), evidencian que para ambos sexos es importante también la práctica de su especialidad atlética para conseguir un fin, ya sea en forma de medallas o marcas en su categoría de edad. Esto queda igualmente evidenciado a la vista de los altos valores obtenidos por hombres y mujeres en su percepción del éxito, muy similares en las dimensiones ego y tarea, como obtuvieron Steinberg et al. (2001) y Zarauz y Ruiz-Juan (2013).

Los elevados niveles obtenidos en motivación intrínseca a las experiencias estimulantes y al logro, satisfacción intrínseca, esfuerzo y habilidad con su práctica deportiva, así como los moderados niveles de motivación extrínseca, percepción del éxito por ego y tarea y los bajos niveles obtenidos en amotivación, aburrimiento y uso de técnicas de engaño, evidencian que el atleta veterano español se man- tiene en la práctica de su especialidad deportiva principalmente por la satisfacción que le produce el simple hecho de ejecutar correctamente los complejos movimientos de su especialidad atlética, reducir tiempos o superar distancias. Por lo tanto, la mejora de sus registros anteriores o volver a hacer un registro que hacía años que no conseguían, es lo que da sentimientos de competencia y logro, más aún si es en competición superando a sus rivales, como explicaban también Steinberg et al. (2001), Da Silva (2009) y Zarauz y Ruiz-Juan (2013).

En la población de veteranos españoles se ha obtenido una relación positiva de la motivación autodeterminada con la dimensión tarea, al igual que Duda et al. (1995), Ferrer-Caja y Weiss (2000) y White y Duda (1994), y también con la dimensión ego, como obtuvieron Kim y Gill (1997). Igualmente positiva ha sido la relación entre la motivación intrínseca y extrínseca, como obtuvieron Moreno et al. (2007). Estas relaciones entre los diferentes niveles de autodeterminación y las diferentes maneras de percibir el éxito reafirman que esta población practica su especialidad atlética principalmente por la satisfacción de hacerlo y lograr buenos registros, pero también les resulta importante la consecución de victorias, medallas o marcas en su correspondiente categoría de edad.

Resulta relevante la relación positiva entre la motivación autodeterminada con la dimensión tarea, la diversión y el esfuerzo, así como de la motivación no autodeterminada con las dimensiones ego, aburrimiento y engaño en los atletas veteranos, como obtuvieron Castillo et al. (2002, 2004), Duda y Nichols (1992), Duda et al. (1995), Ruiz-Juan et al. (2010), Ruiz-Juan y Zarauz (2013) y White y Duda (2004) en sus respectivas poblaciones. Asimismo, la amotivación se relaciona negativamente con diversión y positivamente con aburrimiento y engaño. Esto muestra la importancia de fomentar la motivación intrínseca y la orientación a la tarea para aumentar la diversión y esfuerzo en la práctica deportiva y, por tanto, la adherencia y un mayor mantenimiento en el tiempo de la misma.

A la misma conclusión se puede llegar observando que los atletas con alto SDI muestran mayor orientación a la tarea, diversión y esfuerzo que los de bajo SDI, mientras que estos tienen mayor aburri- 
miento y uso de técnicas de engaño que los de alto SDI. Parece existir una clara relación entre la motivación autodeterminada con la dimensión tarea en esta población, como se obtuvo también en estudios precedentes (Amorose, Anderson-Butcher \& Flesch, 2005; Moreno et al., 2007; Parish \& Treasure, 2003).

Son las mujeres las que obtienen mayor SDI que los hombres, como le sucedió a Ruiz-Juan y Zarauz (2011) en la población de corredores de maratón españoles y a Moreno et al. (2007) y Pelletier et al. (1995) en otras poblaciones de deportistas. En cuanto a la edad, los veteranos más jóvenes muestran mayor SDI y orientación hacia la tarea, al igual que Moreno et al. (2007) o Xiang y Lee (2002).

Según la especialidad atlética practicada, fueron los corredores de resistencia quienes muestran mayor aburrimiento que los especialistas en pruebas de velocidad, saltos o lanzamientos, lo cual puede deberse a la mayor variedad en los sistemas de entrenamiento de estas últimas. Datos similares obtuvieron Ruiz-Juan y Zarauz (2013) con respecto a su ansiedad.

Los atletas veteranos que entrenan más de dos horas al día son quienes más se divierten y esfuerzan y dicen tener una mayor percepción del éxito por ego, lo que contrasta con la percepción del éxito por tarea que obtuvieron los deportistas del estudio de Moreno et al. (2007).

Tener entrenador mostró en los veteranos un mayor SDI, orientación a la tarea, diversión y percepción del éxito por ego y el hecho de entrenar acompañado de otros atletas, fue síntoma de mayor diversión y esfuerzo, como obtuvieron Castañeda y Campos (2012). Esto evidencia la importancia de estar asesorado por un técnico y tener compañeros de entrenamiento, como se obtuvo también en la población de maratonianos españoles por Llopis y Llopis (2006) y Ruiz-Juan y Zarauz (2012b).

A modo de conclusiones, tras lo obtenido en los análisis de varianza y regresivo, podría decirse que para obtener un perfil motivacional que aumente el grado de adherencia y su mantenimiento en el tiempo en la población de veteranos españoles, habría que considerar el fomentar la motivación intrínseca y la orientación a la tarea, en actividades variadas dirigidas por un entrenador, en sesiones con compañeros y de algo más de dos horas, lo cual aumenta el esfuerzo y diversión de los atletas, sobre todo en mujeres.

\section{Referencias}

Amorose, A. J., Anderson-Butcher, D., Flesch, S., \& Klinefelter, L. (2005). Perceived motivational climate and self-determined motivation in male and female high school athletes. Research Quarterly for Exercise and Sport, 76, A96-A97.

Baker, J., Horton, S., \& Weir, P. (2010). The masters athlete: Understanding the role of sport and exercise in optimizing aging. New York: Routledge.

Balaguer Solá, I., \& Atienza, F. (1994). Principales motivos de los jóvenes para jugar al tenis. Apunts, 31(122), 285-299.

Balaguer, I., Atienza, F. L., Castillo, I., Moreno, Y., \& Duda, J. L. (1997, septiembre). Factorial structure of measures of satisfaction/interest in sport and classroom in the case of Spanish adolescents. Trabajo presentado en la Fourth European Conference of Psychological Assessment, Lisboa, Portugal.

Bollen, K., \& Long, J. (1994). Testing structural equation models. Newbury Park, CA: Sage.

Carretero-Dios, H., \& Pérez, C. (2005). Normas para el desarrollo y revisión de estudios instrumentales. International Journal of Clinical and Health Psycho$\log y, 5(3), 521-551$.

Carratalá, E. (2003). Análisis de la teoría de las metas de logro y de la autodeterminación en los planes de especialización deportiva de la Generalitat Valenciana. Tesis Doctoral sin publicar, Facultad de Psicología, Universidad de Valencia, Valencia, España.

Castañeda, C., \& Campos, M. C. (2012). Motivación de los estudiantes de la Facultad de Ciencias de la Educación (Universidad de Sevilla) hacia la práctica de actividad físico deportiva. Retos. Nuevas Tendencias en Educación Física, Deporte y Recreación, 22, 57-61.

Castillo, I., Balaguer. I., \& Duda, J. L. (2002). Las perspectivas de meta de los adolescentes en el contexto deportivo. Psicothema, 14(2), 280-287.

Castillo, I., Balaguer. I., Duda, J. L., \& García-Merita, M. L. (2004). Factores psicosociales asociados con la participación deportiva en la adolescencia. Revista Latinoamericana de Psicología, 36(3), 505-515. 
Cervelló, E. (1996). La motivación y el abandono deportivo desde la perspectiva de las metas de logro. Tesis Doctoral, Universidad de Valencia, Valencia, España.

Cervelló, E., Calvo, R., Ureña, A., Martínez, M., \& Guzmán, J. F. (2006). Situational and dispositional predictors of task involvement in Spanish professional female volleyball players. Journal of Human Movement Studies, 50(1), 47-63.

Cervelló, E., Escartí, A., \& Balagué, G. (1999). Relaciones entre la orientación de meta disposicional y la satisfacción con los resultados deportivos, las creencias sobre las causas de éxito en deporte y la diversión con la práctica deportiva. Revista de Psicología del Deporte, 8(1), 7-19.

Cervelló, E., Hutzler, Y., Reina, R., Sanz, D., \& Moreno, J. A. (2005). Goal orientations, contextual and situational motivational climate and competition goal involvement in Spanish athletes with cerebral palsy. Psicothema, 17(4), 633-638.

Chantal, Y., \& Bernache-Asollant, I. (2003). A prospective analysis of self-determined sport motivation and sportspersonship orientations. Athletic Insight, The Online Journal of Sport Psychology, 5(4). Recuperado de http://www.athleticinsight.com/ Vol5Iss4/Sportspersonship.htm

Chantal, Y., Robin, P., Vernat, J. P., \& Bernache-Asollant, I. (2005). Motivation, sportspersonship, and athletic aggression: A mediational analysis. Psychology of Sport and Exercise, 6(2), 233-249.

Da Silva, E. (2009). Motivaçâo de atletas idosos na associaçâo de veteranos de atletismo do Estado do Rio de Janeiro. Tesis Doctoral, Universidad Niterói, Rio de Janeiro, Brasil.

De Pero, R., Amici, S., Benvenuti, C., Minganti, C., Capranica, L., \& Pesce, C. (2009). Motivation for sport participation in older Italian athletes: The role of age, gender and competition level. Sport Sciences for Health, 5(2), 61-69.

Deci, E. L., \& Ryan, R. M. (1985). Intrinsic motivation and self-determination in human behavior. New York: Plenum Press.

Duda, J. L., Chi, L., Newton, M., Walling, M. D., \& Catley, D. (1995). Task and ego orientation and intrinsic motivation in sport. International Journal of Sport Psychology, 26(1), 40-63.
Duda, J. L., \& Nicholls, J. G. (1992). Dimensions of achievement motivation in schoolwork and sport. Journal of Educational Psychology, 84(3), 290-299.

Ferrer-Caja, E., \& Weiss, M. (2000). Predictors of intrinsic motivation among adolescent students in physical education. Research Quarterly for Exercise and Sport, 71(3), 267-279.

Guivernau, M., \& Duda, J. L. (1994). Psychometric properties of a Spanish versión of The Task and Ego Orientation in Sport Questionnaire (TEOSQ) and Beliefs about the Causes of Success Inventory. Revista de Psicología del Deporte, 5, 31-51.

Hair, J. F., Black, W. C., Babin, B. J., \& Anderson, R. E. (2009). Multivariate data analysis (7th ed.). New York: Pearson Prentice Hall.

Hoyle, R. H. (1995). Structural equation modeling: Concepts, issues, and applications. Thousand Oaks, CA: Sage.

Hu, L., \& Bentler, P. M. (1999). Cutoff criteria for fit indexes in covariance structure analysis: Conventional criteria versus new alternatives. Structural Equation Modeling, 6(1), 1-55.

Iso-Ahola, S., \& St.Clair, B. (2000). Toward a theory of exercise motivation. Quest, 52(2), 131-147.

Kim, B. J., \& Gill, D. L. (1997). A crosscultural extension of goal perspective theory to Korean youth sport. Journal of Sport and Exercise Psychology, 19(2), 142-155.

Kline, R. (1998). Principles and practice of structural equation modeling. New York: Guilford.

Llopis, D., \& Llopis, R. (2006). Razones para participar en carreras de resistencia. Un estudio con corredores aficionados. Cultura, Ciencia y Deporte, 2(4), 33-44.

Losier, G., \& Vallerand, R. (1994). The temporal relationship between perceived competence and self-determined motivation. The Journal of Social Psychology, 134(6), 793-801.

Martens, M. P., \& Webber, S. N. (2002). Psychometric properties of the Sport Motivation Scale: An evaluation with College Varsity Athletes from the U.S. Journal of Sport Eु Exercise Psychology, 24(3), 254-270.

Medic, N., Starkes, J. L. Young, B. W., \& Weir, P. L. (2006). Motivation for sport and goal orientations in Masters athletes: Do masters swimmers differ 
from mastes runners? Journal of Sport and Exercise Psychology, 28, s132.

Medic, N., Starkes, J. L., Young, B. W., Weir, P. L., \& Giajnorio, A. (2004). Masters athlethe's motivation: Evidence of age and gender differences. $\mathrm{Ca}$ nadian Society for Psychomotor Learning and sport Psychology Abstracts, Saskatoon, Saskatchewan.

Medic, N., Young, B. W., Starkes, J. L., Weir, P. L., \& Grove, J. R. (2009). Gender, age, and sport differences in relative age effects among US Masters swimming and track and field athletes. Journal of Sports Sciences, 27(14), 1535-1544.

Moreno Murcia, J. A., Cervelló Gimeno, E., \& GonzálezCutre Coll, D. (2007). Analizando la motivación en el deporte: un estudio a través de la teoría de la autodeterminación. Apuntes de Psicología, 25(1), 35-51.

Moreno Murcia, J. A., Martínez Galindo, C., \& Alonso Villodre, N. (2006). Actitudes hacia la práctica físico-deportiva según el sexo del practicante. Revista Internacional de Ciencias del Deporte, 2(3), 20-43.

Nicholls, J. G. (1984). Achievement motivation: Conceptions of ability, subjective experience, task choice and performance. Psychological Review, 91(3), 328-346.

Núñez Alonso, J. L., Martín-Albo Lucas, J., \& Navarro Izquierdo, J. G. (2007). Propiedades psicométricas de la versión española de la Escala de Motivación Deportiva. Revista de Psicología del Deporte, 16(2), 211-223.

Parish, L. E., \& Treasure, D. C. (2003). Physical activity and situational motivation in physical education: Influence of the motivational climate and perceived ability. Research Quarterly for Exercise and Sport, 74(2), 173-182.

Pelletier, L. G., Fortier, M. S., Vallerand, R. J., Tuson, K. M., Brière, N. M., \& Blais, M. R. (1995). Toward a new measure of intrinsic motivation, extrinsic motivation, and amotivation in sports: The Sport Motivation Scale (SMS). Journal of Sport and Exercise Psychology, 17(1), 35-53.

Real Federación Española de Atletismo. (2012). Comparativa histórica de Licencias de Veteranos. Disponible en http://www.rfea.es/veteranos/licencias.asp

Roberts, G. C., \& Balagué, G. (1989, agosto). The development of a social-cognitive scale in motivation.
Trabajo presentado en el Seventh World Congress of Sport Psychology, Singapur.

Roberts, G. C., \& Balagué, G. (1991, septiembre). The development and validation of the Perception of Success Questionnaire. Trabajo presentado en el Congreso de la FEPSAC, Colonia, Alemania.

Ruiz-Juan, F., Gómez López, M., Pappous, A., Alacid Cárceles, F., \& Flores Allende, G. (2010). Dispositional goal orientation, beliefs about the causes of success and intrinsic satisfaction in young elite players. Journal of Human Kinetics, 26, 123-136.

Ruiz-Juan, F., \& Zarauz Sancho, A. (2011). Validación de la versión española de las Motivations of Marathoners Scales (MOMS). Revista Latinoamericana de Psicología, 43(1), 139-156.

Ruiz-Juan, F., \& Zarauz Sancho, A. (2012a). Predictor variables of motivation on Spanish master athletes. Journal of Human Sport and Exercise, 7(3), 617-628.

Ruiz-Juan, F., \& Zarauz Sancho, A. (2012b). Variables que hacen negativamente adicto a correr al maratoniano español. Retos. Nuevas Tendencias en Educación Física, Deporte y Recreación, 21, 38-42.

Ruiz-Juan, F., \& Zarauz Sancho, A. (2013). Análisis de la ansiedad en el atletismo: un estudio con veteranos. Revista Internacional de Ciencias del Deporte, 33(9), 222-235.

Steinberg, G., Grieve, F. G., \& Glass, B. (2001). Achievement goals across the lifespan. Journal of Sport Behavior, 24(3), 298-307.

Vallerand, R. J. (1997). Toward a hierarchical model of intrinsic and extrinsic motivation. En M. P. Zanna (Ed.), Advances in experimental social psychology (pp. 271-360). New York: Academic Press.

White, S. A., \& Duda, J. L. (1994). The relationship of gender, level of sport involvement, and participation motivation to task and ego orientation. International Journal of Sport Psychology, 25(1), 4-18.

White, S. A., Kavussanu, M., Tank, K. N., \& Wingate, J.M. (2004). Perceived parental beliefs about the causes of success in sport: relationship to athletes' achievement goals and personal beliefs. Scandinavian Journal of Medicine Eु Science in Sports, 14(1), 57-66.

Xiang, P., \& Lee, A. (2002). Achievement goals, perceived motivational climate, and students' self- reported mastery behaviors. Research Quarterly for Exercise and Sport, 73(1), 58-65. 
Zahariadis, P. N., Tsorbatzoudis, H., \& Grouios, G. (2005). The Sport Motivation Scale for Children: Preliminary analysis in physical education classes. Perceptual and Motor Skills, 101(1), 43-54.

Zarauz Sancho, A., \& Ruiz-Juan (2013a). Ansiedad, satisfacción, percepción y creencias sobre las causas del éxito en atletas veteranos españoles. Ansiedad y Estrés, 19(1), 83-93.

Zarauz Sancho, A., \& Ruiz-Juan (2013b). Motivaciones de los maratonianos según variables sociodemográficas y de entrenamiento. Retos. Nuevas Tendencias en Educación Física, Deporte y Recreación, 24, 50-56. 
